\title{
Use of Cosmetic Products and Related Adverse Reactions among Health Science Students
}

\author{
Gbednet Theophilus Kureh ${ }^{1,2 *}$, Arnold Ndesangia ${ }^{2}$, Robert Dickson Opio ${ }^{3}$, Idiongo Okon Umoh', Joshua Ojodale Aruwa ${ }^{5}$, Godwin \\ Aiyabalu Okoruwa ${ }^{6}$ \\ 'Department of Clinical Pharmacology, Faculty of Medicine, St. Francis University College of Health and Allied Sciences, Ifakara, TANZANIA. \\ 2Department of Clinical Pharmacy, Kampala International University in Tanzania, Pugu road, Dar es Salaam, TANZANIA. \\ ${ }^{3}$ Department of Microbiology and Immunology, Kampala International University, Pugu road, Dar es Salaam, TANZANIA \\ ${ }^{4}$ Department of Physiology, Faculty of Medicine, Kampala International University Western Campus, Ishaka, Bushenyi, UGANDA. \\ ${ }^{5}$ Department of Pharmacology and Toxicology, Faculty of Pharmacy, Kampala International University Western Campus, Ishaka, Bushenyi, UGANDA. \\ ${ }^{6}$ Department of Clinical Pharmacy, Faculty of Pharmacy, Kampala International University Western Campus, Ishaka, Bushenyi, UGANDA.
}

\begin{abstract}
Objectives: This research was carried out in Dar es Salaam, Tanzania to assess cosmetic use and related adverse reactions among university students studying medicine and pharmacy in Dar es Salaam, Tanzania. Methods: A cross sectional study design was employed and data was collected by random stratified sampling technique. Data was collected and analyzed using SPSS version 20 and the chi square test was used to determine the associations between variables. Results: Findings indicated that majority $(91 \%)$ of respondents were using cosmetic products at the time of the study. More than half of respondents (58.4\%) admitted to have experienced at least one type of adverse reaction from using cosmetic products. The most commonly experienced adverse reactions were allergic reactions $(30.8 \%)$ followed by burning sensation $(18.5 \%)$ mostly from perfumes and face products. Moreover, there were associations $(p<0.05)$ between use of cosmetic products and some demographic variables (gender and year of study) and there were also associations $(p<0.05)$ between adverse reactions and gender. Findings again revealed that, $17.9 \%$ of the respondents use cosmetics for the purpose of skin protection,
\end{abstract}

$17.9 \%$ s to boost attractiveness and improve beauty. Majority of respondents obtained awareness about the possible adverse effects of cosmetic products from friends and family $(29.5 \%)$, the media $(23.2 \%)$, internet $(21.4 \%)$ and fellow students (17\%). Respondents were also mostly aware of the adverse effects of the cosmetics but still used them. Conclusion: There is a high occurrence and knowledge of cosmetics use- related adverse reactions.

Key words: Cosmetics, Cosmetic products, Cosmetics use-related adverse reactions, Allergic reactions, Skin protection.

Correspondence

Mr. Gbednet Theophilus Kureh,

Department of Clinical Pharmacology, Faculty of Medicine, St. Francis University College of Health and Allied Sciences, P. O. Box 175, Ifakara, TANZANIA.

Phone: +255659135045

Email: theokureh@gmail.com

DOI: 10.5530/jyp.2020.12.74

\section{INTRODUCTION}

The use of cosmetic products dates back to ancient times where people topically applied various substances for reasons ranging from religious rituals to beautification and therapy. ${ }^{1,2}$ Cosmetics are defined as articles intended to be rubbed, poured, sprinkled, or sprayed on, introduced into, or otherwise applied to the human body for cleansing, beautifying, promoting attractiveness or altering appearance. ${ }^{3}$ Common examples of cosmetic formulations described in this definition are skin lotions, perfumes, fingernail polishes, moisturizers, eye shadows, lipsticks and facial makeup preparations, cleansing shampoos, hair colors, permanent waves and deodorants, as well as any substance intended for use as a component of a cosmetic product. The cosmetic industry is fast growing in economic and product value, with the global market estimated to be worth $\$ 863$ billion by $2024 .{ }^{4}$ Although cosmetics can help us feel more beautiful, their use has been associated with many adverse and unwanted reactions generally traced to their main ingredients or excipients. ${ }^{5-7}$ Several studies conducted in Africa, Asia and the Middle East on the awareness, attitude and perception of cosmetic users revealed a high prevalence of cosmetic use especially among young people as well as a high incidence of adverse reactions. ${ }^{8-15}$ Skin bleaching rates among women in Tanzania and other East African countries are estimated to be as high as $30 \%,{ }^{16}$ reasons cited are mainly to improve beauty and attractiveness as dark skinned women are generally regarded inferior in beauty when compared to women of Middle eastern or Asian ancestry. ${ }^{16}$ Another study conducted among students of tertiary institutions in Tanzania reported a high level of awareness of the adverse and unwanted reactions associated with the application of cosmetic products, it also reported high prevalence of the adverse effects as well as high level of cosmetic usage. ${ }^{9}$ Reactions to cosmetics have been observed more in women than men because women tend to use cosmetic products more than men. ${ }^{17,18}$ Unlike pharmaceutical products, cosmetic products have less stringent regulatory requirements in many countries. In the United States for example, there is no clear regulation requiring cosmetic manufacturers to conduct safety and efficacy testing before marketing their products, nor a clear regulation requiring cosmetic manufacturers to release their chemical formulas or to report adverse reactions of their products to the FDA. ${ }^{3}$ This may be a contributing factor to the high prevalence of cosmetic-induced adverse effects especially in developing countries like Tanzania where the level of regulation is generally poor. Cosmetic use-related adverse reactions occur but are generally underreported or overlooked. In a survey conducted by pharmacists involving 4373 consumers in Naples, Italy, $60 \%$ of individuals who reported to have experienced cosmetic use-related adverse reaction did not consider

This is an open access article distributed under the terms of the Creative Commons Attribution-NonCommercial-ShareAlike 4.0 License, which allows others to remix, tweak, and build upon the work non-commercially, as long as the author is credited and the new creations are licensed under the identical terms. 
seeking medical attention. Surprisingly, $2.5 \%$ who sought medical help continued using those products while taking medications to manage the adverse effects. ${ }^{19}$ Similarly, the Norwegian Institute of Public health notes that after its introduction in 2008, the National Register of Adverse Effects from Cosmetic Products received about 442 notifications on cosmetics related adverse effects between 2008 and 2014, majorly reported by women between ages 30-56 and mainly submitted by pharmacists. ${ }^{20}$

An understanding of the prevalence of the adverse reactions arising from cosmetics use among future doctors and pharmacists can form the basis for advocacy, regulation, policy review or health promotion. This research was therefore carried out to determine the cosmetic use pattern and occurrence of cosmetics related adverse effects among medicine and pharmacy students in Kampala International University in Tanzania (KIUT), Dar es Salaam, Tanzania.

\section{METHODOLOGY}

This study was conducted among medicine and pharmacy students in the School of Health Sciences, Kampala International University in Tanzania in Pugu Road, Dar es Salaam, Tanzania. The university offers multidisciplinary undergraduate programs among which are medicine and pharmacy. A sample of 150 respondents was arbitrarily selected from a database of 1354 medicine and pharmacy students at Kampala International University in Tanzania. While recruiting the respondents, the inclusion criteria applied involved the selection of female and male students studying at the university at the time when data was collected. Respondents were randomly selected from each of the stratum (year of study) for medicine (754) and pharmacy (600) students. A total of 150 questionnaires were distributed to the respondents to be collected on a pre-arranged date. A total of 10 questionnaires were discarded due to a high amount of missing data and 28 were not returned by participants leaving 112 questionnaires for analysis.

The questionnaire was designed based on review of literature and its content validated by a group of experts in the Department of Clinical Pharmacy, Kampala International University in Tanzania.

The questionnaire design contained both open and close-ended questions. Participants responded to questions about their demography, whether they used cosmetic products or not, types of cosmetics being used and the reasons, if any, for such use. Participants also indicated the types of adverse reactions (if any) they have experienced in the course of their cosmetic use, the types of cosmetic products that gave rise to such reactions and lastly they were asked if they were aware of possible adverse effects that can arise from the use of cosmetics. They were also asked about their sources of recommendation for the cosmetic products they were using and knowledge about possible adverse reactions associated with cosmetic products.

The study was approved by the Ethical Committee of Kampala International University in Tanzania. Participants were recruited voluntarily and administered the questionnaire after an informed verbal consent and were assured that the information they provided would be treated with anonymity and confidentiality.

The responses from the questionnaires were coded into SPSS version 20 (Chicago IL) and then used to generate descriptive statistics and these were represented in figures, percentages and bar chat. Associations between variables were analyzed using the $\chi^{2}$ test.

\section{RESULTS}

A total of 112 participants responded to the administered questionnaire, 28 participants did not return their questionnaires while 10 of the returned questionnaires were discarded due to high amount of missing data. Of the 112 questionnaires analyzed, $71 \%$ are females and $36.6 \%$ are males. Up to $91 \%$ of the respondents attest to using cosmetic products. The age with highest use of cosmetics was ages $20-24$, accounting for $61.6 \%$ of respondents followed by the 25-29 years of age which accounted for $15.2 \%$ of the analyzed questionnaires (Table 1 ). There were associations $(p<0.05)$ between use of cosmetic products and some demographic variables (gender and year of study) as well as associations $(p<0.05)$ between cutaneous adverse reactions and gender (Table 2). The study also revealed little difference between medicine students and pharmacy student's participation in the study and on their use of cosmetic products. Majority of the respondents $(37.5 \%)$ use cosmetics for the purpose of skin protection, followed by $17.9 \%$ who use cosmetics to boost attractiveness and improve beauty respectively. Other factors include boosting confidence, following trend and fashion and using the beneficial ingredients in cosmetics (Table 3).

Most of the respondents (58.04\%) admitted experiencing at least one adverse reactions to cosmetics. The most common adverse reaction was allergic reactions reported by $30.8 \%$ of the respondents followed by

Table 1: Demographic characteristics of respondents.

\begin{tabular}{ccc}
\hline Variable & & Frequency (\%) \\
\hline Gender & Male & $41(36.6)$ \\
& Female & $71(63.4)$ \\
Age & $15-19$ & $6(5.4)$ \\
& $20-24$ & $69(61.6)$ \\
& $25-29$ & $17(15.2)$ \\
& $30-34$ & $9(8.0)$ \\
Year of study & 35 above & $11(9.8)$ \\
& First year & $21(18.8)$ \\
& Second year & $51(45.5)$ \\
Course of study & Third year & $38(33.9)$ \\
& Fourth year & $2(1.8)$ \\
& Medicine & $58(51.8)$ \\
& Pharmacy & $54(48.2)$ \\
\hline
\end{tabular}

Table 2: Proportion of respondents that use cosmetics and those that experienced adverse reactions.

\begin{tabular}{|c|c|c|c|c|c|}
\hline 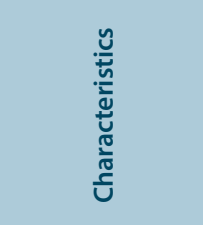 & $\begin{array}{l}\frac{0}{0} \\
\frac{0}{\frac{0}{2}} \\
\frac{5}{7}\end{array}$ & 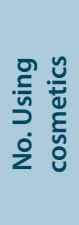 & $\frac{0}{\frac{2}{0}}$ & 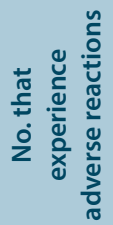 & $\begin{array}{l}\frac{0}{2} \\
\frac{2}{10} \\
\frac{1}{2}\end{array}$ \\
\hline \multirow[t]{2}{*}{ Gender } & Male & 31 & 0.0083 & 19 & 0.00004 \\
\hline & Female & 71 & & 46 & \\
\hline \multirow[t]{5}{*}{ Age in years } & $15-19$ & 4 & 0.1556 & 3 & 0.05952 \\
\hline & $20-24$ & 65 & & 44 & \\
\hline & $25-29$ & 15 & & 9 & \\
\hline & $30-34$ & 7 & & 6 & \\
\hline & 35 and above & 11 & & 3 & \\
\hline \multirow[t]{4}{*}{ Year of Study } & First year & 21 & 0.0020 & 15 & 0.4066 \\
\hline & Second year & 50 & & 34 & \\
\hline & Third year & 29 & & 15 & \\
\hline & Fourth year & 2 & & 1 & \\
\hline \multirow[t]{2}{*}{ Course of Study } & Medicine & 51 & 0.2270 & 36 & 0.14941 \\
\hline & Pharmacy & 51 & & 29 & \\
\hline
\end{tabular}

Significant at $p<0.05$ 
Kureh, et al.: Cosmetics use-related Adverse Reactions among Students

Table 3: Factors influencing the use of cosmetics.

\begin{tabular}{cc}
\hline Factor & Frequency (\%) \\
\hline Skin protection & $42(37.5)$ \\
Trend and fashion & $9(8.0)$ \\
Boost attractiveness & $20(17.9)$ \\
Improve beauty & $20(17.9)$ \\
Boost confidence & $19(17.0)$ \\
Beneficial ingredients & $2(1.8)$ \\
\hline
\end{tabular}

Table 4: Type of adverse reactions experienced.

\begin{tabular}{cc}
\hline Adverse reaction & Frequency (\%) \\
\hline Skin rash & $11(16.9)$ \\
Burning sensation & $12(18.5)$ \\
Allergic reaction & $20(30.8)$ \\
Itching & $9(13.8)$ \\
Eczema & $5(7.7)$ \\
Skin lighting & $5(7.7)$ \\
Others & $3(4.6)$ \\
\hline
\end{tabular}

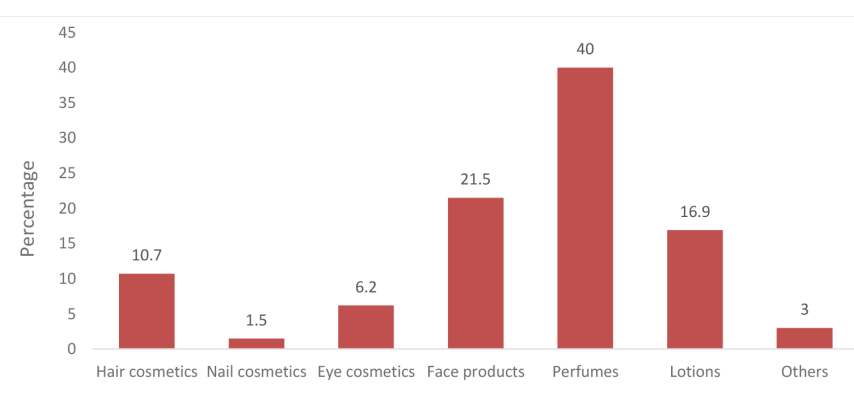

Figure 1: Cosmetic products associated with adverse reactions.

burning sensation in $18.5 \%$ of the respondents; skin rash and itching also accounted for $16.9 \%$ and $13.8 \%$ respectively, while skin lightening and eczema accounted for $7.7 \%$ of adverse reactions respectively. Other reactions accounted for $4.6 \%$ among respondents. These revealed that majority of the respondents experience allergic reactions followed by burning sensations (Table 4). The cosmetics identified for causing the most adverse reactions were: perfumes in $40 \%$ of respondents, face products in $21.5 \%$ of respondents, lotions $16.9 \%$ of respondents and hair cosmetics in $10.7 \%$ (Figure 1). Majority (29.5\%) of the respondents obtained awareness about the adverse effects associated with cosmetic use from friends and family, $23.2 \%$ from the media, $21.4 \%$ from internet, $17.0 \%$ from fellow students and $8.9 \%$ from other sources (Table 5)

\section{DISCUSSION}

The results obtained revealed that, majority of the respondents (91.1\%) were using cosmetics at the time of this study. These results indicate a widespread use of cosmetic as almost all respondents in the study use cosmetics irrespective of gender. A similar observation was also reported in a similar study ${ }^{8}$ where $85 \%$ of the respondents were using at least one type of cosmetics and skin rash was the predominant adverse reaction experienced by the respondents. Similar studies conducted in Ethiopia and Malaysia report skin rash to be the most common unwanted effect among participants. ${ }^{8,13}$ This indicates that cosmetics use related adverse reactions occur significantly among cosmetics users but are generally underreported.
Table 5: Source of Awareness on induced adverse effects.

\begin{tabular}{cc}
\hline Source & Frequency (\%) \\
\hline Fellow students & $19(17.0)$ \\
Friends and Family & $33(29.5)$ \\
Media & $26(23.2)$ \\
Internet & $24(21.4)$ \\
Others & $10(8.9)$ \\
\hline
\end{tabular}

Other studies ${ }^{14,16}$ revealed that, $97.8 \%$ and $97.3 \%$ of participants respectively had a habit of using cosmetics. That the respondents with the highest percentage of cosmetic use are young adults between ages 20-24 is suggestive of future increase in use of cosmetics from continuous use by current users and likely initiation especially of the younger population growing into the age bracket. The use of cosmetics mostly for skin protection $(37.5 \%)$ and boosting attractiveness $(17.9 \%)$ as seen in this study corroborates those of a study done in Iran. ${ }^{11,12}$ Results also revealed that $53.6 \%$ of the respondents admit to have experienced adverse reaction from using cosmetics compared to $39.3 \%$ that did not experience any adverse reaction. This almost double higher occurrence of adverse reactions should serve as a call for regulatory authorities to enforce more stringent regulatory measures as the predicted growth of the number of cosmetic users ${ }^{4}$ will most likely result in increased incidence of adverse reactions associated with cosmetic use. Allergic reactions are the most commonly experienced adverse reactions followed the feeling of a burning sensation. This may be due to fragrance added in cosmetic products which studies suggest account for a high prevalence of allergic reactions among users.?

It is also interesting to note that perfumes, face products and lotions users reported the highest incidence of adverse reactions-in descending frequency (Figure 1). Studies have revealed that fragrance in perfumes and other cosmetic products account allergic reaction in up to $1 \%$ of the general population. ${ }^{7}$ Only recently, "cosmetovigilance", a term used to describe surveillance on the safety of cosmetics is beginning to receive global attention. ${ }^{21}$ For example, the Norwegian National Register of Adverse Effects from Cosmetic Product was opened in 2008 and received submissions from only healthcare professionals, it has received far more submissions from 2015 when it was made accessible to the public. A prevalence of this magnitude (53.6\%) should serve as a call to regulatory authorities to create avenues for cosmetic surveillance and appropriate reporting of cosmetics userelated adverse reactions.

A comparatively higher percentage of the respondents (69.6\%) were aware of the adverse effects of cosmetics but still used them. This finding is consistent with other research ${ }^{9,10}$ which revealed that respondents were aware of the number of adverse reactions associated with the use of cosmetic products but continued to use them. This suggests a compelling desire for improved beauty and attractiveness even with substantial knowledge of the health consequences. This is also similar to the pattern observed in the study conducted in Naples, Italy. ${ }^{19}$

The sources of awareness about the adverse effects was found to be predominantly from family and friends (29.5\%) followed by the media $(23.2 \%)$ while $21.4 \%$ obtained information from the internet, $17 \%$ from fellow students and $8.9 \%$ from other sources. These results revealed that, awareness is mostly obtained from family, friends and the media. Health advocacy and the formulation of health policies and a thorough implementation of these policies will benefit the society. An intensive government supported sensitization of the possible and common adverse effects associated with the use of cosmetic products especially through media campaigns, proper education of healthcare personnel who would serve in adding 
strength in the advocacy as well as empowering regulatory authorities such as the Tanzania Food and Drugs Authority (TFDA) technically and through the law will help curb the rising incidence of adverse reactions associated with the use of cosmetic products.

\section{Limitation}

This study was based on respondents' self-reported experience of adverse effects from cosmetic product use. Therefore, there is a probability of reporting incorrect reactions and reports may be subject to respondents' bias. Another limitation is the small sample size involved in this research.

\section{CONCLUSION}

This study has therefore demonstrated a use of cosmetic products among students of in the health sciences as well as a high prevalence of adverse effects associated with the use of these products. Allergic reaction was most commonly experienced by the students from the use of perfumes, face products and lotions. Students had a high level of awareness about adverse effects from cosmetic products which was mostly obtained from family and friends and the media.

\section{ACKNOWLEDGEMENT}

The authors thank lecturers of Department of Clinical Pharmacy, Kampala International University in Tanzania for their support.

\section{CONFLICT OF INTEREST}

The authors declare that they have no conflicts of interest

\section{ABBREVIATIONS}

FDA: United states Food and Drug Administration; KIUT: Kampala International University in Tanzania; TFDA: Tanzania Food and Drug Agency; SPSS: Statistical Package for Social Sciences.

\section{REFERENCES}

1. Chaudhri SK, Jain NK. History of cosmetics. Asian J Pharm Sci. 2009;3(3).

2. Eze UC, Tan C, Yeo AL. Purchasing Cosmetic Products: A Preliminary Perspective of Gen-Y. Contemp Manag Res. 2012;8(1):51-60.

3. US Food and Drug Administration. FDA Authority Over Cosmetics: How Cosmetics Are Not FDA-Approved, but Are FDA-Regulated. Available from: https://www.fda.gov/Cosmetics/GuidanceRegulation/LawsRegulations/ ucm074162.htm
4. Zion Market Research. Global Cosmetic Products Market Will Reach USD 863 Billion by 2024. Available from: https://www.globenewswire.com/newsrelease/2018/06/22/1528369/0/en/Global-Cosmetic-Products-Market-WillReach-USD-863-Billion-by-2024-Zion-Market-Research.html

5. Johansen JD, Rastogi SC, Menne T, Johansen JD. Threshold responses in cinnamic-aldehyde-sensitive subjects: Results and methodological aspects. Contact Derm. 1996;34(3):165-71.

6. Schafer T, Bohler E, Ruhdorfer S, Weigl L, Wessner D, Filipiak B, et al. Epidemiology of contact allergy in adults. Allergy. 2001;56(12):1192-6.

7. DeGroot AC, Frosch PJ. Adverse reactions to fragrances: A clinical review. Contact Derm. 1997:36(2):57-86.

8. Binega G, Tegegne GT, Gelaw BK, Defersha MG. Assessment of cosmetic use and its skin reaction among post graduate students in university of Gondar, Gondar, North East Ethiopia. Int J Cur Res Chem Pharm Sci. 2014;1 (7):08-1217.

9. Massoro ZZ. Information Seeking and use Behaviour on Beautification products among Students of Higher Learning Institutions. Middle-East J Sci Res. 2016;24(1):149-56.

10. Tejal P, Nishad D, Amisha J, Umesh G, Desai KT, Bansal RK. Cosmetics and health: usage, perceptions and awareness. Bangladesh $\mathrm{J}$ Med Sci. 2013;12(4):392-7.

11. Jalilian F, Ahmadpanah M, Karimi M, Salehi I, Vahidinia A, Emdadi S. Prevalence and reasons for cosmetic products use among female students in Hamedan Universities. Int J Dermatol Cosmetic. 2012;3(1):9-15.

12. Hosseini SN, Mari AM, JouybariTA, Salehi I, Vahidinia AA, Emdadi S. Cosmetic Products Use Intention among Iranian Female College Students. Int Sci Index. $2014 ; 7$.

13. Mansor N, Ali DE, Yaacob MR. Cosmetic usage in Malaysia: understanding of the major determinants affecting the users. Int J Business Social Sci. 2010;1(3).

14. Meharie BG, Ambaye AS, Haimanot YM. A cross-sectional study on assessment of cosmetics utlization and self-reported adverse reactions among Wollo University, Dessie campus female students. Dessie, North East Ethiopia. Eur J Phar Med Res. 2014;2(2):49-63.

15. Dibaba H, Yadesa D, Legesse B, Shewamene Z. Cosmetics utilization pattern and related adverse reactions among female university students. Int J PharmSci Res. 2013;4(3):997.

16. Lewis KM, Harris S, Camp C, Kalala W, Jones W, Ellick KL, et al. The historical and cultural influences of skin bleaching in Tanzania. In The Melanin Millennium. Springer, Dordrecht. 2013;19-38. Available from: https://link.springer.com/chapter/1 0.1007/978-94-007-4608-4_2

17. Schnuch A, Geier J, Uter W, Frosch PJ, Lehmacher W, Aberer W, et al. National rates and regional differences in sensitization to allergens of the standard series: Population-adjusted frequencies of sensitization (PAFS) in 40,000 patients from a multicenter study (IVDK). Contact Derm. 1997;37(5):200-9

18. Rees JL, Friedmann, Matthews JNS. Sex differences in susceptibility to dinitrochlorobenzene (DNCB). Br J Dermatol. 1989;120(3):371-4.

19. DiGiovanni C, Arcoraci V, Gambardella L, Sautebin L. Cosmetovigilance survey: Are cosmetics considered safe by consumers?. Pharmacol Res. 2006;53(1):16-21.

20. Norwegian Institute for Public Health. Statistics from the National Register of Adverse Effects from Cosmetic Products. Available from: https://www.fhi.no/ en/el/cosmetic-allergy/statistics-from-the-national-regist/

21. Toklu HZ, Antigua $A$, Lewis $V$, Reynolds $M$, Jones J. Cosmetovigilance: A review of the current literature. J Family Med Prim Care. 2019;8(5):1540-5. 